\title{
Severe haemorrhage from a lumbar artery as a complication of percutaneous renal biopsy
}

\author{
M.H. Jamison ${ }^{1}$ and R.A. Coward ${ }^{2}$

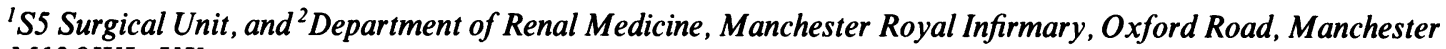 \\ M139WL, UK
}

\begin{abstract}
Summary: A patient is described with severe haemorrhage from a lumbar artery following percutaneous renal biopsy. The exact source of haemorrhage in this previously unreported complication proved difficult to locate even at surgical exploration.
\end{abstract}

\section{Introduction}

Percutaneous renal biopsy is a generally safe procedure with complications occurring in $8.1 \%$ of patients and a $1.4 \%$ incidence of clinically apparent perirenal haematomas (Diaz-Buxo \& Donadio, 1975). The site of bleeding in previous reports has been from the renal vasculature; we report the first published case of severe haemorrhage from a lumbar artery.

\section{Case report}

A 57 year old retired train driver with a 2 y history of Type II diabetes mellitus, proliferative diabetic retinopathy and glaucoma presented with persistent vomiting. Investigations confirmed a diagnosis of IgG kappa type multiple myeloma with a serum paraprotein band, and $50 \%$ plasma cells in the bone marrow. Serum urea was $11.3 \mathrm{mmol} / \mathrm{l}$, and creatinine $146 \mu \mathrm{mol} / 1$. He was nephrotic with $5.9 \mathrm{~g} / 24 \mathrm{~h}$ of proteinuria with urine electrophoresis demonstrating albumin and IgG kappa paraprotein.

A renal biopsy was performed to exclude amyloid, and define if the renal lesion was related to his diabetes or myeloma. Prior to biopsy blood pressure was $160 /$ $85 \mathrm{~mm} \mathrm{Hg}$ and coagulation tests were normal apart from a prolonged thrombin time (45 s, control $25 \mathrm{~s}$ ). The lower pole of the left kidney was biopsied by an experienced operator (RAC), with a Franklin modification of the Vim Silverman needle under image intensification, using standard techniques and obtaining two renal cores, with no clinical problems during the procedure.

M.H. Jamison, F.R.C.S. and R.A. Coward, M.R.C.P. Correspondence: M.H. Jamison, S5 Unit, Manchester Royal Infirmary, Oxford Road, Manchester M139WL.

Accepted: 23 November 1983
Six hours afterwards the patient complained of pain and became clinically shocked with an expanding haematoma in the left loin. An emergency, intravenous urogram showed a normally functioning right kidney but the left kidney was displaced medially and obstructed by a soft tissue swelling. Twenty hours after the biopsy with continued clinical evidence of bleeding and after a total of 11 units of blood, 4 packs of platelets and 4 units of fresh frozen plasma the left kidney was explored via a loin incision excising the 12th rib. Haematoma of at least four litres was found extending retroperitoneally from the diaphragm to the pelvis. The kidney was fully mobilized and the two puncture sites, oozing a little blood, were identified in the lower pole but no obvious source of massive haemorrhage was located. The peritoneal cavity contained no blood, and in the absence of active bleeding the wound was closed with drainage. Three hours after surgery the loin was re-explored because of continued bleeding from the drains, requiring a further 8 units of blood. After extensive dissection and retraction of the quadratus lumborum muscle the source of haemorrhage was indisputably identified as a lumbar artery which was oversewn, the patient making a satisfactory recovery.

The renal biopsy showed changes of both diabetes and myeloma but staining for amyloid was negative.

\section{Discussion}

The risk of major haemorrhage after renal biopsy is small but significant such that the procedure should only be carried out if the other kidney is adequate, and there is no haemorrhagic tendency - judged by a platelet count of $10 \times 10^{10} / 1$ and a prothrombin time of $<16$ s (McGonigle \& Sharpstone, 1980). Hyperten-

(C) The Fellowship of Postgraduate Medicine, 1985 
sion and small renal size are relative contraindications. Our patient, apart from a prolonged thrombin time secondary to the nephrotic state, fulfilled these criteria for a safe biopsy.

In a series of 1000 consecutive renal biopsies from the Mayo Clinic $8.1 \%$ of patients developed complications including haematuria (69 patients) and perirenal haematomas (14 patients), yet only two patients required surgical exploration and evacuation of the haematoma. Small clinically undetectable perirenal haematomas are undoubtedly more common than this with an incidence of $85 \%$ being recorded using computed tomographic scanning (Rosenbaum et al., 1978).

Although numerous complications of renal biopsy (septicaemia, renal abscesses, perforation of a hollow viscus and arterio-venous fistulae) have been recorded (Kark et al., 1958; Slotkin \& Madsen, 1962) bleeding from a lumbar artery has not. The upper three pairs of

\section{References}

DIAZ-BUZO, J.A. \& DONADIO, J.V. (1975). Complications of percutaneous renal biopsy: an analysis of 1000 consecutive biopsies. Clinical Nephrology, 4, 223.

HOLLINSHEAD, W.H. (1971). Anatomy for Surgeons Vol. 2, 2nd edition, 580. Harper \& Row, New York, Evanston, San Francisco, London.

KARK, R.M., MUERCKE, R.C., POLLAK, V.E., PIRANI, C.L. \& KIEFER, J.H. (1958). An analysis of five hundred percutaneous renal biopsies. Archives of Internal Medicine, 101, 439.

McGONIGLE, R. \& SHARPSTONE, P. (1980). Kidney biopsy. British Medical Journal, 1, 547.

ROSENBAUM, R., HOFFSTEN, P.E., STANLEY, R.J. \& KLAHR, S. (1978). Use of computerised tomography to diagnose complications of percutaneous renal biopsy. Kidney International, 14, 87.

SLOTKIN, E.A. \& MADSEN, P.O. (1962). Complications of renal biopsy: incidence in $\mathbf{5 0 0 0}$ reported cases. Journal of Urology, 87, 13. lumbar arteries usually pass behind the quadratus lumborum muscles (Hollinshead, 1971) and thus are at 3 risk from the renal biopsy needle penetrating the lower $\stackrel{\Phi}{\complement}$ pole, but not revealed when the kidney is exposed $C$ through a standard loin incision to explore a haematoma. This fact accounts for the difficulty in $\stackrel{5}{?}$ locating the bleeding site at the first operation. Further $?$ preoperative investigation by selective renal angiogra- $\frac{\bar{O}}{\bar{c}}$ phy would have excluded the kidney as the site of the $\vec{\sigma}$ haemorrhage but is unlikely to have demonstrated a $\propto$ bleeding lumbar artery unless picked up on preliminary aortic views.

\section{Acknowledgements}

We would like to thank Dr I.W. Delamore for allowing us to report this case and Mrs Y. Banner for typing the manus-.cript. 